\title{
Study of the electrophysical properties of nanostructured porous germanium as a promising material for electrodes of electrochemical capacitors
}

\author{
Nikolay Platonov ${ }^{1, *}$, Nail Suleimanov ${ }^{1,2}$, and Valery Bazarov ${ }^{2}$ \\ ${ }^{1}$ Kazan State Power Engineering University \\ ${ }^{2}$ Zavoisky Physical Technical Institute, FRC Kazan Scientific Center of RAS
}

\begin{abstract}
Electrochemical capacitors (ECC) are a fast charging devices, with high power density, capacity and increased life time. Nanostructured semiconductors are now considered as the promising materials for electrodes of such devices due to its conductive properties and effective surface. One of such materials is the porous germanium which can be used as an electrode in electrochemical capacitors. In this article the novel approach based on the method of ion implantation was developed to grow these structures. This method allows to obtain a structures up to $1 \mu \mathrm{m}$ thick. The object of this work was the investigation of the electrophysical characteristics of samples of nanostructured porous germanium $(\mathrm{Ge})$ depending on the implantation dose and surface morphology. The scientific novelty of this research lies in the search the structures with the highest effective surface area and electronic conductivity, capable of multiplying the energy capacity and specific power of ECC. Methods: The samples of amorphous Ge were grown on dielectric single-crystal substrates of Al2O3. The thickness of samples was 600 and $1000 \mathrm{~nm}$. The magnetron sputtering and ion implantation methods were used to growth these structures. The irradiation with $\mathrm{Ge}+$ ions produced with an energy of $40 \mathrm{keV}$ and the range of implantation doses varied from $2 \cdot 10^{16}$ to $12 \cdot 10^{16}$ ion $/ \mathrm{cm}^{2}$. The study of electrical properties was carried out on the Hall installation HL55PC at the NPP KVANT in Moscow. The following parameters were measured: the sheet concentration of carriers in the near-surface layer, electrical resistance, mobility of the charge carriers, Hall coefficient. As a result, the dependences of carriers concentration and their mobility as the function of the implantation dose and thickness of the samples of nanostructured porous germanium were determined, and the results were analyzed. Results: It was found that ion implantation of single-crystal germanium leads to an increase in the carrier concentration in the near-surface layer. To sum up, the most suitable material as an electrode for ECC is the porous germanium with the maximum dose of ion implantation and the largest thickness. The maximum sheet carrier concentration that was obtained in the study for $\mathrm{Ge}$ is $10^{17} \mathrm{~cm}^{-2}$.
\end{abstract}

\section{Introduction}

Electrochemical capacitors (supercapacitors) by their properties are located between electrochemical batteries and ordinary electric capacitors $[1,2]$. The main requirements for these energy sources are the high specific power, energy capacity, service life (number of charge-discharge cycles).

In hybrid electrochemical capacitors the accumulation of charge is carried out by two processes: accumulation of Faraday charge as a result of redox reactions on one electrode and the appearance of the capacity of the electric double layer (DES) on the other electrode [3]. ECC are the fast-charging devices with high power density. The decisive factor for achieving a high specific energy content are the surface area of the electrodes and availability of active components for the redox reactions. In this regard, highly porous conducting materials are essential for the manufacture of electrodes of ECCs [4]. All of the above requirements and characteristics of ECCs indicate the need to search of new semiconductor and carbon materials with high electrophysical characteristics. Therefore, the research of new promising materials for electrodes of supercapacitors is an urgent task.

The main task of this study is to find the most optimal morphology of the porous germanium structure, with the best electrophysical characteristics as an electrode in ECC: with a high sheet concentration and mobility of charge carriers.

\section{Literature Review}

The nanostructured semiconductor materials based on germanium $(\mathrm{Ge})$ are considered as the most promising materials for electrodes, along with carbon nanosystems. In this regard, special attention is attracted to approaches based on the development of methods for obtaining

\footnotetext{
* Corresponding author: nickiplatonov@gmail.com
} 
nanostructured porous germanium, with a large number of voids and pores, providing a high effective surface with high electronic conductivity. The high electronic conductivity of porous germanium is due to the small band gap of $\mathrm{Ge}(\mathrm{Eg}=0.66 \mathrm{eV}$ at $300 \mathrm{~K})$. For comparison, this value for silicon ( $\mathrm{Si}$ ) is $1.12 \mathrm{eV}$ at 300 K.

There are several methods to growing porous layers of germanium: thermal deposition (resistive thermal evaporation, electron beam evaporation, etc.), gas deposition methods, deposition from liquid solutions (electrochemical etching) [6].

In treatise [7] are provided a new design of germanium electrodes containing three-dimensional spherical formations of crystalline nanowires on strong one-dimensional nanostructures. These structures were directly grown on a metal current collector by thermal evaporation of germanium.

It is known that high-dose ion implantation into germanium, as a rule, leads to intensive nanopore formation. Implantation of heavy chemically active elements $\left(\mathrm{m}_{\mathrm{ion}}>\mathrm{m}_{\mathrm{Ge}}\right)$ leads to the formation of an ultradispersed phase. In this phase, the surface layers consist of germanium nanoparticles $(10-100 \mathrm{~nm})$. This structure named a highly porous medium [8].

Ion implantation affects the electronic and atomic structure of the crystal when accelerated ions hit on solid surface [9]. Such as a result of the process of ion implantation, in crystal structure are created the radiation defects (violation of the periodic structure of the crystal state). After ion implantation process, the surface can be amorphized, but in our case, the irradiated surface has amorphous properties after magnetron sputtering [10].

In papers $[11,12]$ are considered the main mechanisms of defect formation during ion implantation. An incident accelerated ion during deceleration into structures dislocate the entire groups of atoms from their primary places, which leads to the appearance of disordered regions in the lattice. After irradiation, in the near-surface region of amorphous Ge-films are formed the vacancy complexes and nanopores. Their dimensions gradually increase up to $200 \mathrm{~nm}$ with increasing of dose implantation [13]. In this case, nanopores on the surface lose their upper shell and become visible, as a result of ion sputtering. Also, the results of scientific work [13] show, that nanopores in the porous structure of germanium are distributed throughout the active layer up to its border with the substrate.

Undoubtedly, the question of the effect of radiation dose ion implantation on the concentration and mobility of carriers take a great interest for practical applications.

In treatise [14] was investigated the dependence of the layer concentration of electrons on the dose of $\mathrm{B}^{+}$, $\mathrm{Ag}^{+}, \mathrm{P}^{+}, \mathrm{In}^{+}, \mathrm{Au}^{+}$ions implanted in $\mathrm{n}-\mathrm{CdHgTe}$ with an energy of $150 \mathrm{keV}$. This study showed that for any type of implanted ions, the value of the layer concentration $\left(\mathrm{N}_{\mathrm{s}}\right)$ weakly depends on their dose: at ion doses of $10^{12}$ $10^{15} \mathrm{ion} / \mathrm{cm}^{2}$ the layer concentration of electrons changes in the range of $10^{13}-10^{14} \mathrm{~cm}^{-2}$. Also, the fact that the layer concentration of electrons $\mathrm{N}_{\mathrm{s}}$ does not depend on the type and dose of implanted ions was shown in [15]: the implantation ions of $\mathrm{B}^{+}, \mathrm{Al}^{+}, \mathrm{P}^{+}, \mathrm{Ar}^{+}$into $\mathrm{CdHgTe}$ with an energy of 100-300 keV with a dose of $10^{13}-10^{15}$ ion $/ \mathrm{cm}^{2}$ led to a constant value $\mathrm{N}_{\mathrm{s}}=(5-6) \cdot 10^{14} \mathrm{~cm}^{-2}$, and the electron mobility was $\mu_{\mathrm{n}}=(1-3) \cdot 10^{2}$ $\mathrm{cm}^{2} \cdot \mathrm{V}^{-1} \cdot \mathrm{s}^{-1}$.

At the same time, the value of the layer concentration of electrons increased as a result of ion implantation. The authors of the work assumed that the emerging electronic conductivity in the layers is associated with electrically active radiation defects arising from ion implantation [16].

The saturation of the concentration of charge carriers in the structure occurs at certain doses of implanted ions, depending on their mass. It was shown in [17], that when the concentration of electrons in layers saturates with an increase of the ion dose, the mobility of carriers remains constant value at the level $\mu_{\mathrm{n}}=(3-5) \cdot 10^{3} \mathrm{~cm}^{2} \cdot \mathrm{V}^{-1} \cdot \mathrm{s}^{-1}$ (higher electron mobility for small doses). In [18] was established the opposite fact - with an increasing the dose implantation of $\mathrm{B}^{+}$ions, the effective electron mobility decreased to the value $\mu_{\mathrm{n}}=1.1 \cdot 10^{4} \mathrm{~cm}^{2} \cdot \mathrm{V}^{-1} \cdot \mathrm{s}^{-1}$. In [16] and [18], the electron mobility differs from the almost an order of magnitude. Thus, the clear dependence of the mobility of charge carriers on the implantation dose wasn't found in many experiments. This dependence is multifactorial and specific for each of the materials.

\section{Materials and methods}

Six (6) samples with dimensions of 10x10 mm were prepared for the study (fig. 4). A single-crystal germanium substrate GDG-45 with orientation (111) with thickness $500 \mu \mathrm{m}$ was taken as a zero - reference sample, which was not subsequently subjected to ion implantation. This sample is called "reference sample" for subsequent comparison of the results of ion implantation other samples.

The other five (5) samples were grown by magnetron sputtering: the amorphous Ge layers with different thicknesses $(600 \mathrm{~nm}$ and $1000 \mathrm{~nm})$ were obtained on single-crystal dielectric substrates $\mathrm{Al}_{2} \mathrm{O}_{3}$. At the next stage of preparation, these samples were irradiated with $\mathrm{Ge}^{+}$ions with an energy of $40 \mathrm{keV}$ in the range of implantation doses: $2 \cdot 10^{16} \div 12 \cdot 10^{16}$ ion $/ \mathrm{cm}^{2}$. A dielectric substrate was used to exclude an unnecessary contribution to the conductivity of investigated layer during measurements of the study. As already indicated, a single-crystal sapphire plates $\mathrm{Al}_{2} \mathrm{O}_{3}$ with orientation (012) were used as a substrates. The substrates were cleaned before growth in several stages: on the first stage of preparation the chemical solvents with ultrasound were used, and at the final stage the cleaning in a vacuum chamber by ion etching was provided.

The initial $\alpha$-Ge films with a thickness of $600 \mathrm{~nm}$ and $1000 \mathrm{~nm}$ were obtained by magnetron sputtering of a germanium target $(99.95 \%$ purity, OOO Girmet) in an ultrahigh vacuum setup (SPECS / BESTEC, Germany) at room temperature in an argon atmosphere. The base pressure in the magnetron chamber was no more than $5 \cdot 10^{-9}$ mbar, the working pressure of argon was $6 \cdot 10^{-3}$ mbar. The magnetron power during deposition was 50 
$\mathrm{W}$. The germanium deposition rate was $8.33 \mathrm{~nm} / \mathrm{min}$ and was controlled by a quartz thickness gauge. The thickness of the films was measured with a stylus nanoprofilometer BRUKER Dektak XT [13].

Implantation with $\mathrm{Ge}^{+}$ions was carried out in the dose range $(2-12) \cdot 10^{16} \mathrm{ion} / \mathrm{cm}^{2}$ with an energy of $\mathrm{E}=40 \mathrm{keV}$ and a current density in the ion beam of 4-5 $\mu \mathrm{A} / \mathrm{cm}^{2}$ on an ILU-3 ion accelerator at a residual vacuum of $10^{-5}$ Torr.

The structure of the five (5) investigated samples, which were subjected on ion implantation, is shown in Table 1:

Table 1. Structure of the studied samples.

\begin{tabular}{|c|c|c|c|}
\hline \multirow{4}{*}{$\begin{array}{c}\text { Investigated } \\
\text { layer }\end{array}$} & $\begin{array}{c}\text { Number of } \\
\text { sample, № }\end{array}$ & $\begin{array}{c}\text { Thickness of } \\
\text { porous Ge, } \\
\mathbf{n m}\end{array}$ & $\begin{array}{c}\text { Dose of } \\
\text { ilmplantation, } \\
\mathbf{1 0}^{\mathbf{1 6}}, \mathbf{i o n} / \mathbf{c m}^{\mathbf{2}}\end{array}$ \\
\cline { 2 - 4 } & 1 & 600 & 2 \\
\cline { 2 - 4 } & 2 & 600 & 6 \\
\cline { 2 - 4 } & 4 & 1000 & 4 \\
\cline { 2 - 4 } & 5 & 1000 & 8 \\
\hline Substrate & \multicolumn{3}{|c}{ Single crystal dielectric substrate $(500 \mu \mathrm{m}) \mathrm{Al}_{2} \mathrm{O}_{3}$} \\
\hline
\end{tabular}

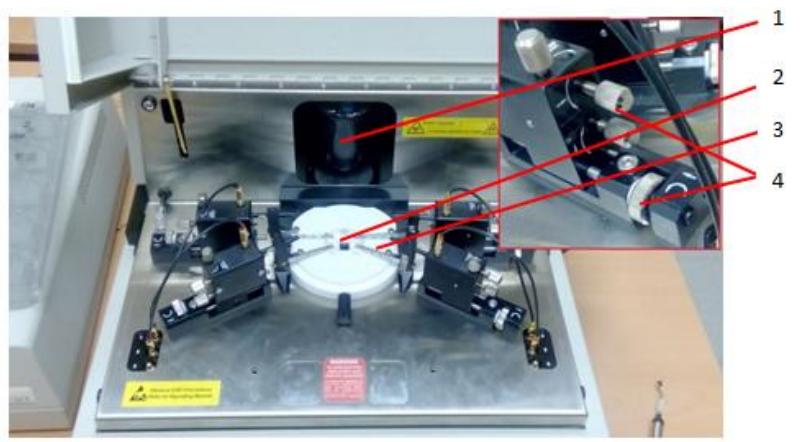

Fig. 1. The Hall installation HL55PC (1 - magnet (to create a magnetic field); 2 - a table for a sample; 3 - contact needles (for passing electric current through the plate); 4 - handles for precise positioning of contacts on the sample).

In this method a big emphasis is paid to the quality of the ohmic contacts. The measurements were performed by means of the four measuring gold needles. Each pairs of contacts are burnt by a pulse current before experiments. If, after reinstalling the quality of contact pairs was not enough the contact points were scratched out with a needle or InGa paste is applied to improve the contact points. In accordance with experimental needs the contact needles should be installed on the sample, so the symmetry factor should be close to 1 . An example of a correctly installed sample is shown in Fig. 2. The measurement parameters for the HL55PC installation for each of the samples are listed in Table 2.
Table 2. Hall Measurement Basic Parameters.

\begin{tabular}{|c|c|c|c|c|}
\hline $\begin{array}{c}\text { Number } \\
\text { of } \\
\text { sample, } \\
\text { No }\end{array}$ & $\begin{array}{c}\text { Magnetic } \\
\text { induction } \\
\text { B, Tesla }\end{array}$ & $\begin{array}{c}\text { Temper } \\
\text { ature T, } \\
\mathbf{K}\end{array}$ & $\begin{array}{c}\text { Measureme } \\
\text { nt current I, } \\
\boldsymbol{\mu} \text { A (DC) }\end{array}$ & $\begin{array}{c}\text { Applicatio } \\
\text { n of InGa } \\
\text { paste }\end{array}$ \\
\hline $\begin{array}{c}\text { Referen } \\
\text { ce } \\
\text { sample }\end{array}$ & 0.332 & 273 & 0.1 & no \\
\hline $\mathbf{1}$ & 0.332 & 273 & 0.1 & no \\
\hline $\mathbf{2}$ & 0.332 & 273 & 0.3 & yes \\
\hline $\mathbf{3}$ & 0.332 & 273 & 20.0 & yes \\
\hline $\mathbf{4}$ & 0.332 & 273 & 0.07 & yes \\
\hline $\mathbf{5}$ & 0.332 & 273 & 28.0 & no \\
\hline
\end{tabular}

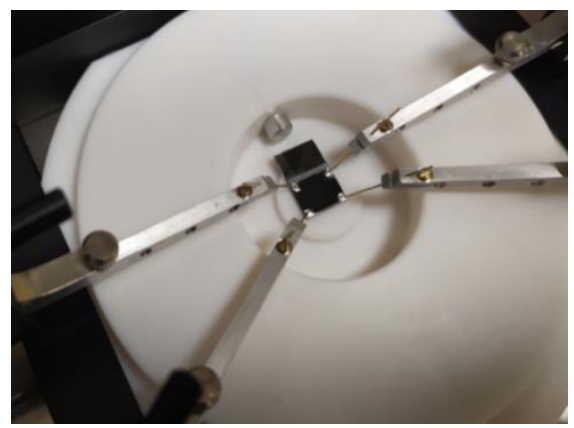

Fig. 2. Correctly installed sample in HL55PC.

Each of the samples was measured several times: in 4-5 different positions (after each measurement, the sample was rotated by $90^{\circ}$ ). The total result of Hall measurements is given as the average of 4-5 measurements

\section{Results and discussion}

In the Table 3 the results of measurements of electrophysical characteristics of the nanostructured porous germanium samples are presented. Since germanium belongs to semiconductors of mixed type of conductivity, the modulus (absolute value) of the layer concentration of the charge carriers and Hall coefficient are given in the resulting table.

According to the data in Table 3 the sheet concentration of the charge carriers in the "reference" sample is tens times less than in the samples that were subjected to ion implantation and on the contrary, the mobility and Hall coefficient are several times higher. Based on the obtained data were plotted the following dependences: sheet concentration and mobility of free charge carriers in the studied layers of porous $\mathrm{Ge}$ depending on the implantation dose (Fig. 3 and 4) and the thickness of the samples (Fig. 5). 
Table 3. Measurement results.

\begin{tabular}{|c|c|c|c|c|c|}
\hline $\begin{array}{c}\text { Number of } \\
\text { sample, № }\end{array}$ & $\begin{array}{c}\text { Dose of } \\
\text { implantation, } \\
\mathbf{1 0}^{\mathbf{1 6}}, \mathbf{i o n} / \mathbf{c m}^{\mathbf{2}}\end{array}$ & $\begin{array}{c}\text { Thickness of } \\
\text { investigated } \\
\text { layer }\end{array}$ & $\begin{array}{c}\text { Sheet concentration } \\
\text { of charge carriers } \\
\mathbf{N}_{\mathbf{s}} \mathbf{\mathbf { c m } ^ { - 2 }}\end{array}$ & $\begin{array}{c}\text { Mobility of } \\
\mathbf{c h a r g e} \text { carrier, } \\
\mathbf{c m}^{\mathbf{2}} \cdot \mathbf{V}^{\mathbf{- 1}} \cdot \mathbf{s}^{\mathbf{- 1}}\end{array}$ & $\begin{array}{c}\text { Hall coefficient } \\
\mathbf{R}_{\mathbf{H}}, \mathbf{m}^{\mathbf{3}} / \mathbf{C}\end{array}$ \\
\hline $\begin{array}{c}\text { Reference } \\
\text { sample }\end{array}$ & - & $500 \mu \mathrm{M}$ & $1.44 \cdot 10^{11}$ & 15.30 & 4330 \\
\hline $\mathbf{1}$ & 2 & $600 \mathrm{~nm}$ & $6.22 \cdot 10^{12}$ & 0.76 & 235 \\
\hline $\mathbf{2}$ & 6 & $600 \mathrm{~nm}$ & $4.69 \cdot 10^{12}$ & 2.07 & 138 \\
\hline $\mathbf{3}$ & 4 & $1000 \mathrm{~nm}$ & $1.79 \cdot 10^{14}$ & 0.30 & 4 \\
\hline $\mathbf{4}$ & 8 & $1000 \mathrm{~nm}$ & $6.95 \cdot 10^{12}$ & 2.12 & 110 \\
\hline $\mathbf{5}$ & 12 & $1000 \mathrm{~nm}$ & $1.51 \cdot 10^{17}$ & 0.16 & 0.04 \\
\hline
\end{tabular}

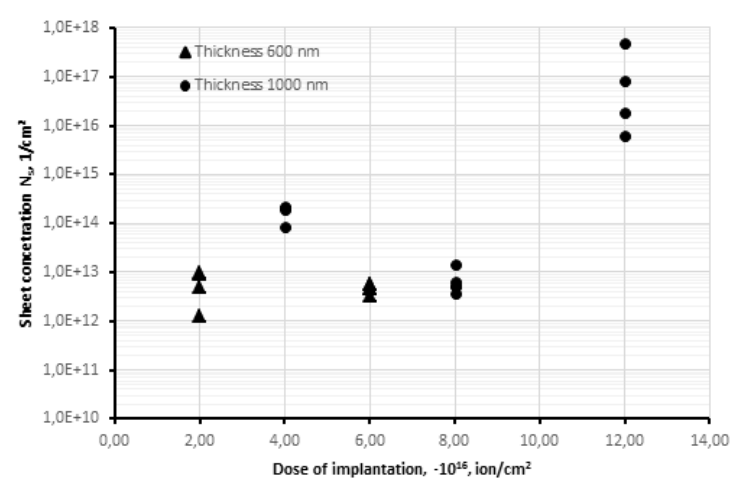

Fig. 3. Dependence of the sheet concentration on the ion implantation dose.

In samples No. 1 and No. 2 with a thickness of 600 $\mathrm{nm}$ and different doses of implantation, the concentration of charge carriers is kept at approximately the same level (Fig. 3). For samples No. 3, 4, 5 with a thickness of 1000 $\mathrm{nm}$, according to the graph in Figure 3, there is a tendency to an increase in the sheet concentration of carriers with an increase the implantation dose.

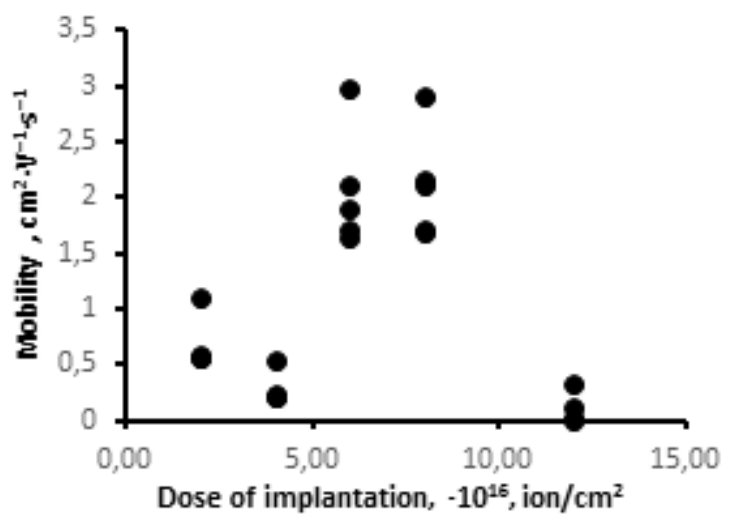

Fig. 4. Dependence of mobility of charge carriers on the implantation dose.

In samples No. 1 and No. 2 with a thickness of 600 $\mathrm{nm}$ and different doses of implantation, the concentration of charge carriers is kept at approximately the same level (Fig. 3). For samples No. 3, 4, 5 with a thickness of 1000 $\mathrm{nm}$, according to the graph in Figure 3, there is a tendency to an increase in the sheet concentration of carriers with an increase the implantation dose.
Analyzing the graphs in Figures 3 and 5, it should be noted that the maximum sheet concentration of charge carriers $\left(10^{16} \div 10^{17} \mathrm{~cm}^{-2}\right)$ is achieved in sample No. 5 which was grown with the maximum implantation dose $\left(12 \cdot 10^{16}\right.$ ion $\left./ \mathrm{cm}^{2}\right)$ and having the maximum thickness $(1000 \mathrm{~nm})$. Since amorphous Ge after ion implantation becomes porous with a large number of defects that increase the electron layer conductivity [9]. The thickness of the sample also affects its properties, albeit to a lesser extent, which we can see in Fig.5: with increasing thickness of the samples, the concentration of charge carriers growing too.

Investigating the experimental data of mobility charge carries, according to the graph in Fig. 4, it should be noted: with an increasing the implantation dose, the values of mobility first increased (samples No. 2 and No. 4) from 1.64 to $3 \mathrm{~cm}^{2} \cdot V^{-1} \cdot \mathrm{s}^{-1}$, and then decreased to the minimum value $0.16 \mathrm{~cm}^{2} \cdot \mathrm{V}^{-1} \cdot \mathrm{s}^{-1}$ (sample No. 5).

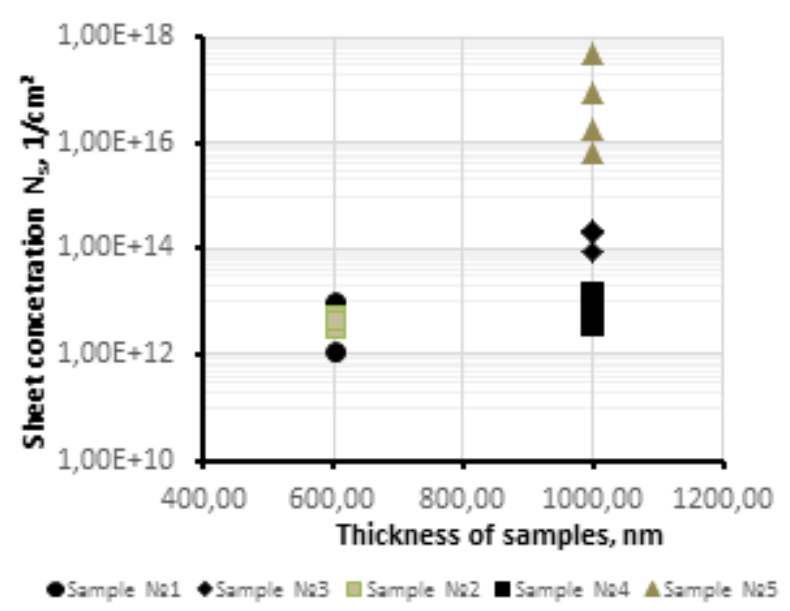

Fig. 5. Dependence of layer concentration on sample thickness.

The obtained dependences of the sheet concentration of charge carriers in the layers under study is in agreement with the results of [20], in which it was found that the concentration of charge carriers increases with an increase in the implantation dose. At the same time, in [14] and [15], it was found that the layer concentration of electrons Ns does not depend on either the type or the dose of implanted ions. Note that in the cited works, various ions with different masses were used.

Similar to the results of our research, in [13] it was shown that as a result of ion implantation, the irradiated 
material gradually becomes porous and the pore size increases with increasing doses. As noted in the literature review, ion implantation with large doses leads to the appearance of a large number of point, linear, and bulk crystal defects (pores, channels). In addition to pores and vacancies, dislocations, grain boundaries, channels and their associations arise in the material.

Ions of Ge are considered heavy and in accordance with [20], and upon hitting the Ge surface form dislocation defects in porous germanium even at low implantation doses. The increasing the electron concentration in such structures may be connected with appearance of defects, as claimed the authors of [21]. They assumed that the presence of radiation defects leads to n-type conductivity of irradiated materials. Thus, it was concluded that samples with a higher implantation dose have a greater number of radiation defects, which enhance the surface conductivity of the layer.

Undoubtedly, there is a possibility that in this study we did not "catch" the range of implantation doses where concentration of radiation defects saturates, as shown in [22-25]. So, for samples No. 1 and No. 2 with a thickness of $600 \mathrm{~nm}$, the expected change in the layer concentration did not occur when the implantation dose was changed. In this regard, it can be assumed that the saturation of the concentrations of charge carriers for different thicknesses of the samples will occur at different doses of implantation.

Comparing the values of the mobility of charge carriers with other works it can be noted that the value of mobility obtained by measuring our samples is less then in treatises [16] and [18]. This fact can also be associated with the presence of a large number of defects in the structure, which "absorb" free electrons and holes.

\section{Conclusions}

1.The measurements of the electrophysical characteristics of porous amorphous Ge (obtained by the method of ion implantation) is carried out: with different doses of $\mathrm{Ge}^{+}$ion implantation and the thickness of the working layer.

2. It was found that ion implantation of single-crystal germanium leads to the amorphization and an increase in the concentration of carriers in the surface layer.

3. The maximum concentration of carriers is observed at the maximum sample thickness $(1000 \mathrm{~nm})$ and implantation dose is $12 \cdot 10^{16} \mathrm{ion} / \mathrm{cm}^{2}$ and consist $1.5 \cdot 10^{17} \mathrm{~cm}^{-2}$.

4. It has been suggested that at a certain dose of implantation, the surface layer is saturated with charge carriers, but this value may differ for layers of different thicknesses - this requires additional research.

5. It has been established that one of the possible reasons for the increased concentration of carriers and low mobility in the material under study, can be structural defects which was appeared during irradiation materials with $\mathrm{Ge}^{+}$ions.

6. A large effective surface area and the maximum charge carrier concentration is expected for the samples of porous germanium with the maximum dose of ion implantation and the largest thickness as an electrode for ECC.

The reported research was funded by Russian Foundation for Basic Research and the Council of Ministers of the Tatarstan Republic, grant № 18-48-160027.

\section{References}

1. I. Varakin, Electrochemical capacitors for hybrid electric vehicles, 9th international seminar on double layer capacitors and similar storage: abstracts, reports, Florida, USA (1999)

2. I. Varakin, et all, New ultracapacitors developed by the joint-stock company "ESMA" for various applications, International seminar on capacitors with a double layer and similar storage: abstracts, Dokl., Florida (1998)

3. S. Hadzi-Jordanov, H. Angerstein-Kozlowska, M. Vikovic, B. Conway, J. Electrochem. Soc., 125, 1471 (1978)

4. V. Shurygin, Supercapacitors, Assistants or Potential Competitors to Battery Power Supplies, Electronics: Science, Technology, Business, 3, 20-24 (2003)

5. N. Choudhary, C. Li, J. Moore, et all, Asymmetric Supercapacitor Electrodes and Devices (2017)

6. L. Dunyushkina, Introduction to methods of producing film electrolytes for solid oxide fuel cells. Russian Academy of Sciences, Ural Branch, Institute of High-Temperature Electrochemistry, Yekaterinburg (2015)

7. Gwang-Hee Lee, S. Joon Kwon, Kyung-Soo Park, et all, Germanium microflower-onnanostem as a high-performance lithium ion battery electrode, Scientific Reports, 4, 6883 (2014)

8. G. Zakirov, I. Khaibullin, M. Zaripov, Formation of an ultrafine layer of germanium during ion bombardment, FTP, 17 (2), 232-234 (1983)

9. M. Kumakhov, Energy losses and ion ranges in solids (BSU Publishing House, 320, 1979)

10. M. Ibragimova, B. Khaibullin, N. Sainov, Transformation of the crystal structure of $\mathrm{Cd}_{\mathrm{x}} \mathrm{Hg}_{1-}$ xTe upon ion implantation, FTP, 27 (8), 12761281 (1993)

11. A. Baranov, L.S. Smirnova (Ed.), Annealing of complex defects in irradiated semiconductors (Novosibirsk: Nauka, 224, 1979)

12. A. Baranov, L.S. Smirnova (Ed.), Accumulation of defects and amorphization processes during bombardment of semiconductors with ions (Novosibirsk: Science, 23-60, 1979)

13. N. Lyadov, T. Gavrilova, S. Khantimerov, et all, Pore formation in thin films of germanium upon implantation of $\mathrm{Ge}+$ ions, Technical Physics Letters, 46 (14) (2020)

14. S. Margalit, Y. Nemirovsky, I. Rotstein, Electrical properties ion-implanted layers in 
$\mathrm{Hg}_{0.79} \mathrm{Cd}_{0.21} \mathrm{Te}$, J. Appl. Phys., 50 (10), 6386-6389 (1979)

15. A. Kolodny, J. Kidron, Properties of ionimplanted junction in mercury-cadmium-telluride, IEEE Transact. on Electr. Devices, V. ED-27, 1, 37-42 (1980)

16. G.L. Destefanis, Ion implantation in $\mathrm{Hg} 1-$ xCdxTe, Nucl. Instr. Meth., 209/210, 567-580 (1983)

17. V. Vasiliev, V. Ovsyuk, N. Talipov, Thermal stability of photodiodes based on p-type CdxHg1xTe heteroepitaxial layers, Abstracts of the Meeting "Actual problems of semiconductor photoelectronics", Photonics-2003, Novosibirsk, 65 (2003)

18. K. Boltar, V. Korolkov, A. Frolov, Investigation of the electrophysical characteristics of ionimplanted layers CdxHg1-xTe, VIII All-Union symp., Narrow band gap semiconductors and semimetals, Lvov, 2, 14-16 (1991)

19. L. Van der Pauw, A method of measuring the resistivity and Hall coefficient on lamellae of arbitrary shape, Philips Technical Review: journal, 20, 220-224 (1958)

20. S. Kozyrev, L. Vodopyanov, Formation of radiation defects in $\mathrm{Hg}_{0.8} \mathrm{Cd}_{0.2} \mathrm{Te}$ during ion implantation, Implantation of ions AG + FTP, 17 (5), 893-899 (1983)

21. L. Bubulac, W. Tennant, S. Shin, et all, Ion implantation study of $\mathrm{HgCdTe}$, Jap. J. Appl. Phys., 19, 495-500 (1980)

22. N. Talipov, Physico-technological bases of doping of narrow-gap semiconductor compounds CdxHg1-xTe by radiation-thermal effects, Dissertation for the degree of Doctor of Physics and Mathematics, FGAOU "National Research Tomsk State University", Tomsk (2015)

23. G. Bahir, R. Kalih, Structure of ion-implanted and annealed $\mathrm{Hg} 1-\mathrm{xCdxTe}$, J. Appl. Phys., 54 (6), 3129-3140 (1983)

24. A. Voitsekhovsky, A. Kokhanenko, Distribution of profiles of radiation defects in MCT after ion implantation, Applied Physics, 4, 38-44 (2000)

25. L. Magel, T. Sigmon, Dose rate effects in indiumimplanted $\mathrm{Hg}_{1-\mathrm{x}} \mathrm{Cd}_{\mathrm{x}} \mathrm{Te}, \mathbf{8 6}, 756-761$ (1988). 